\title{
Design and Development to Face Recognition System by Synthesizing AADHAR Enabled Platform for Online Voting
}

\author{
Nitesh, Gaurav Kumar, Rohit Kaushik, Krishan Kumar, Vivek Birla
}

\begin{abstract}
It's a web based named as "online voting system" which aims to develop a computerized system to make the election process more secure and user friendly. This system has many features which are generally not available in current days. With the help of this system, user can cast their vote without going to any polling booth; it can save their time and effort. This system can also save money of government which is spent in preparation of election process. This system uses face detection and recognition technology to make this system more secure and safe. Overall this project of ours is being developed to help staff of Election commission of India and also reduce the human efforts.
\end{abstract}

KEYWORDS- Online voting; Biometric authentication; Security system

\section{INTRODUCTION}

Election plays an important role in such a huge democratic country like India where the leader is elected by resident. Elections keep a fair nation functioning, as they give individuals the option to choose their own government. So the election must be a free and fair process.

\section{Manuscript received May 23, 2020}

Nitesh, Department of Computer Science, Amity University, Gurugram, Haryana, India India (e-mail: nitesh2802@outlook.com).

Gaurav Kumar, Department of Computer Science, Amity University, Gurugram, Haryana, India, ,India

Rohit Kaushik, Department of Computer Science, Amity University, Gurugram, Haryana, India,

Krishan Kumar, Assistant Professor, Department of Computer Science, Amity University, Gurugram, Haryana, India

Vivek Birla, Assistant Professor, Department of Computer Science, Amity University, Gurugram, Haryana, India
Every citizen of a democratic country has a right of voting with his/her own choice. One of the fundamental issues in the conventional democratic framework is that it expends bunches of labor and resources. Also a few people may include in unlawful course of action during this process of election or its preparation.There are some disadvantages of the conventional election voting process which is being used in our country such as machine stops working, chances of brutality, time consuming, resource consuming, spot arranged etc. Many people couldn't vote because the voter has to reach the poll booths to vote or some people like those who are living far away from their origin birth place where they are allowed to vote. So to get rid of there drawbacks, a new System is introduced i.e. Online Voting System, which provides accuracy, security, flexibility, mobility etc. An online voting System in a web based application to use in election process. Initially ballot paper technique was used in election process. Then Electronic Voting Machine comes, these are easy to store the data and easily manageable. These are more secure than the ballot paper and less time consuming. Now, we proposed system with biometric authentication to make voting process more secure and reduce the time taken in voting process. By using this, voter can cast their vote for their favourite candidate by using their system. We use Face detection and Recognition Technology for authentication of voter that he/she is the correct user or not. We provide many modules in which admin can login in the system and monitor the various operation. Also user can login in the system and use their right to vote. When the Voter uses the system, the system will capture his/her image using web camera \& try to match with the image stored in database. If both images are same then voter can cast his/her vote. Most higher learning establishments in Kenya conduct elections rroutinely to elect an understudy leadership for choose them. They proposed the process of an online system, which includes systems like enlistment of voters, vote casting, vote checking, and pronouncing results which would establish a decent answer for substitute of framework that is in the institutes in Kenya.

\section{LITERATURE REVIEW}

Shrivastava et al.[1] build an online voting system in which voter can cast their vote without going to pooling booths. They use username and passwords for authentication and do not use biometric authentication. In 
our project we uses biometric authentication, which can help to improve the security of the system.

Abdulhamid et al. [2] build E-voting system to make the voting process more efficient. They use username and secret word to confirm the client and furthermore validation of meeting and meeting break has been given to forestall meeting capturing in our undertaking we use Face Recognition Technology to make sure about the framework from unapproved client.

Firas.I.Hazzaa proposed a "Web Based Voting System Using Fingerprint Design and Implementation"(Hazzaa et al., 2012)[3] to improve the security of voting system and make it more secure. The anticipated EVS allows the electorate to investigate their unique mark that is facilitated with the current duplicate put away in the database.

Nautiyal [4] based on the face recognition process under propose system this work has suggested better approach for identification used in criminal identification. They used Image partitioning algorithm. In this work pre captured image was taken in future win 10 to take a real image and perform real time image processing.

Patel \& Patel[5] paper presents the face acknowledgment technique dependent on incomplete Hausdorff separation. They utilized Hausdorff separation. Further work should be possible to improve the time taken by the calculation. This should be possible by pre changing the database pictures and furthermore utilizing a few models to have them into gatherings.

Yamini et al. [6] build class attendance system in Python language using OPENCV, Haar's Cascade and Viola- Jones framework. In universities and cooperate offices, RFID scanners and biometric fingerprints systems are used to mark the attendance of the employees. By using these systems securities and ethics problems occurs. So they build a system using face detection and recognition technique. They used ViolaJones algorithm for face detection and Histogram of Oriented Gradients and Local Binary Patterns for face recognition. And used OpenCV library as image processing framework. This system is much more secure than the previous one and it is less time consuming as we compare it with RFID scanners.

Soomro \& Ali [7] build an authentication application using face recognition technology. They used NI MyRIO and LabVIEW, combination of software and hardware devices integration of OpenCV and NI VISION tool kit to recognize the faces in minimum time. The MyRIO devices is used to process the large real time data with the help of some algorithms which are implemented in it and it is programed through LabVIEW. Also LabVIEW is used to develop the database of the system. To make electronic voting system more secure they used this system for authentication.

Kavitha, S. N. (n.d.).[8]Developed a fully biometric authentication system for voting purpose. They used FM220 Starttek Scanner to scan the human finger prints and store the data. Normal camera is used to capture the image of the voter and PCA(Principal Component Analysis) is used to feature extraction and IRRIS detection. When all the recorded data successfully matched with the Aadhar card data which is saved in the database voter is allow to use the system. They used three layer biometric authentication technique for security of the system which makes it more secure and on the other hand it makes the system more complex.

Face acknowledgment by a PC is modified by python over OpenCV and this paper and this paper is about execute the Eigenfaces and Fisherfaces customized in Python. Geometric component and marker guide utilized toward assemble highlight vector. Calculations utilized for a neighborhood include extraction are Gabor Wavelets, Discrete Cosinus Transform and Local Binary Patterns(Wagner, 2012)[9].

Current voting process have many faults and the objective of this project is to minimize possibilities of manipulation. This project worked on implementation of fingerprint and facial recognition for vote verification. They used different technologies and tools like Python Development Environment, Linux Interfacing Engine and, Visual Basic. This dual authentication system reduces the chances of fraud and illegal activities and improves the security and efficiency of the voting process(P et al., 2018)[10].

\section{EXISTING SYSTEM}

Progressing casting a ballot framework is the system dependent on polling form machine where voters should make their choice by squeezing the catch in machine which is having various images of various candidates. There are numerous security issues like machine got harmed or the individual may make their choice ordinarily or burglary may happen. In this framework, readiness is begun numerous days prior to the political decision which requires cash and labor. Voters need to reach to the surveying corner to give their vote subsequent to remaining in line for quite a while which is very tedious.

\section{PROPOSED SYSTEM}

In this framework we make a thought of web based democratic framework utilizing Biometric discovery for face. In this framework the voter's face is distinguished, clicked and putted away in the database at that point coordinate the clicked picture with the picture present in database to perceive the person. In the event that the two pictures coordinate, voter can give his/her vote to their preferred chosen one. This will be all the more making sure about, dependable, adaptable and less exhausting.

\section{CONTRIBUTIONS}

We address the following contribution of this article:

- We purpose an advance system which helps in voting process and make it more secure from the current system.

- We make this system user friendly, by using this there is no need to go to any pooling booth and don't wait for their number to cast their vote. This system reduces the time complexity and provides a user friendly environment.

- We present a system that is economically feasible, this system only requires a good internet and a mobile or computer system with a web camera. 
- We use biometric authentication for security purpose. Face Recognition technology is used to verify the image of voter from the database.

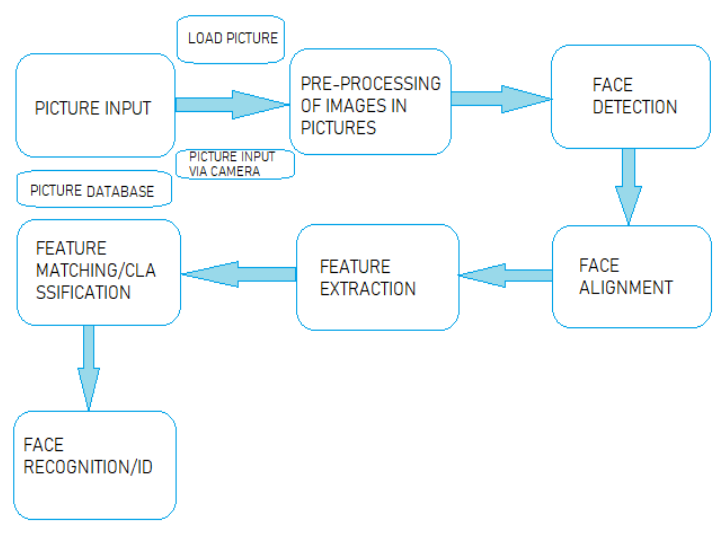

Fig 1: Block diagram of image processing

\section{FUNCTIONAL DESCRIPTION}

Functional description of various software and hardware components is given below:

\section{A. OpenCV}

OpenCV stands for open source Computer Vision Library. This library helps to process an image. It plays a crucial part. It is mainly used to do all the operations related to mages. It plays a major role in real-time operation which is important in today's system. By using it, one can process image \& videos to identify objects, faces or even handwriting of a human.

\section{B. HTML}

It's short for Hypertext Markup Language. It causes us to make electronic pages or records that are shown on World Wide Web. It is a standard markup language for site pages. HTML components are spoken to by <> labels. In this venture, we have make a few layouts through HTML.

\section{CSS}

It's a language that portrays the method of HTML record. CSS hold a ton of errand and limit the format of various site pages. It can control the design of different website pages at the same time. A CSS remarks start with/* and end with*/.

\section{Javascript}

JavaScript is a customer scripted language of HTML and the web. It is independent language created in Netscape. It is utilized when a site page is to be made dynamic and include enhancements pages like rollover, rollout and so forth in HTML, JavaScript code is embedded among $<$ script $>$ and $<$ /script $>$ labels.

\section{E. PostgreSQL}

PostgreSQL, otherwise called Postgres, is a free and open-source social database the board framework underlining extensibility and SQL consistence. We used PostgreSQL maintain all the data. It can easily store and share the data when the system need it.

\section{F. Web camera}

A webcam is a camcorder that takes care of a picture or video progressively to a PC system, for example, the Internet. Webcams are regularly little cameras that sit on a work area, join to a client screen, or are incorporated with the equipment. We can just utilize our PC front camera to catch the picture of a voter.

\section{FACE RECOGNITION WORKING MECHANISM}

\section{A. Face Recognition}

Face or facial acknowledgment is a method of perceiving a human face through innovation. This innovation serves to recognizing or confirming an individual from a computerized picture or a video outline from a video source. A face acknowledgment framework utilizes biometric to delineate highlights from a photo or video. It contrasts the data and a database of realized appearances to discover a match. This framework utilizes PC calculations to choose explicit, unmistakable insights concerning people face.

\section{B. Face detection techniques}

Face discovery is a difficult undertaking since varieties in scale, area, direction, present, outward appearance, impediment, and lighting conditions change the general appearance of countenances. Different methodologies for face identification have been proposed which incorporate, information based, and appearance-based techniques, and through layout coordinating, skin shading, movement, and so on., to make reference to a couple. The face detection process is an essential step as it detects and locates human faces in images and videos.

- The face acknowledgment process is a fundamental development as it perceives and discovers human faces in pictures and chronicles.

- The face get process changes simple data(face) into advanced data(information) in light of person's facial features.

- The face coordinate methodology checks if the two faces are of same person.

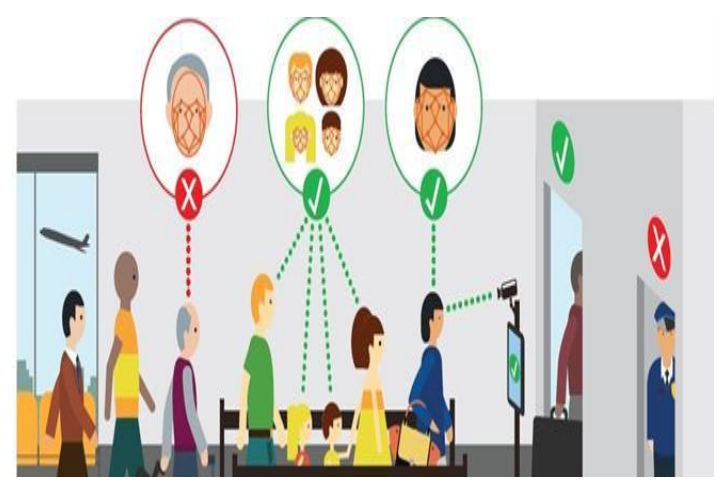

Fig 2: Face recognition and detection system 


\section{Face Recognition using Python}

Python is world's fastest growing programming language. It is object oriented programming language to develop a software or an application and also used in Data analysis, web application, mobile application etc. We have used OpenCV library of Python in our project for recognition and detection of the face of voter.

\section{WORKING MECHANISM}

We add several modules in this system like registration, login, adding political party and result. All modules are interconnected with each other.

\section{A. Registration}

This is registration page, here voter can fill his/her information about their name, gender etc. by the clicking on Create New Voter button, data will store in the database.

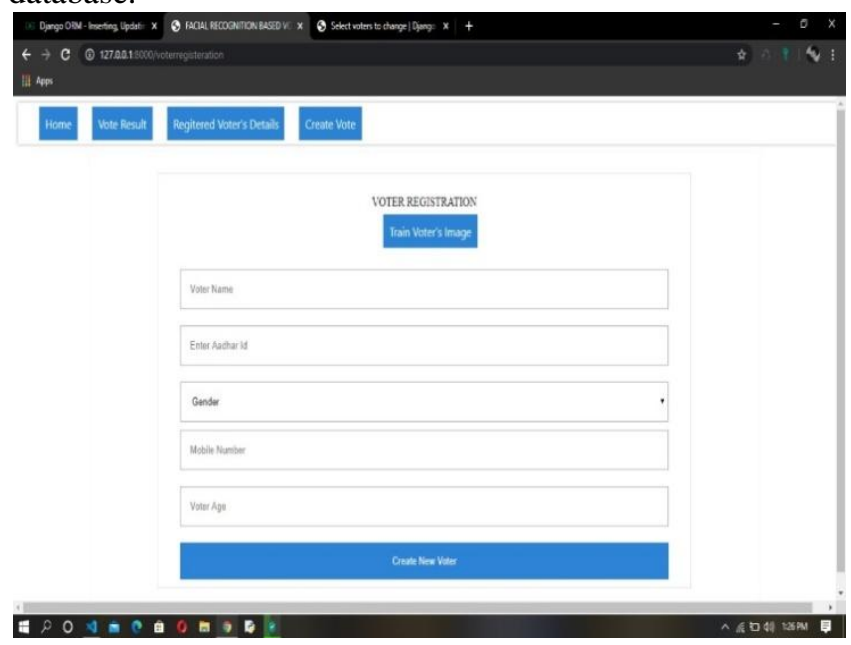

Fig 3: Registration Page

\section{B. Add Political Party}

This is kind of register page, where admin can add or register new political party in the system. Only admin can add the political party name and can upload the logo of the party.

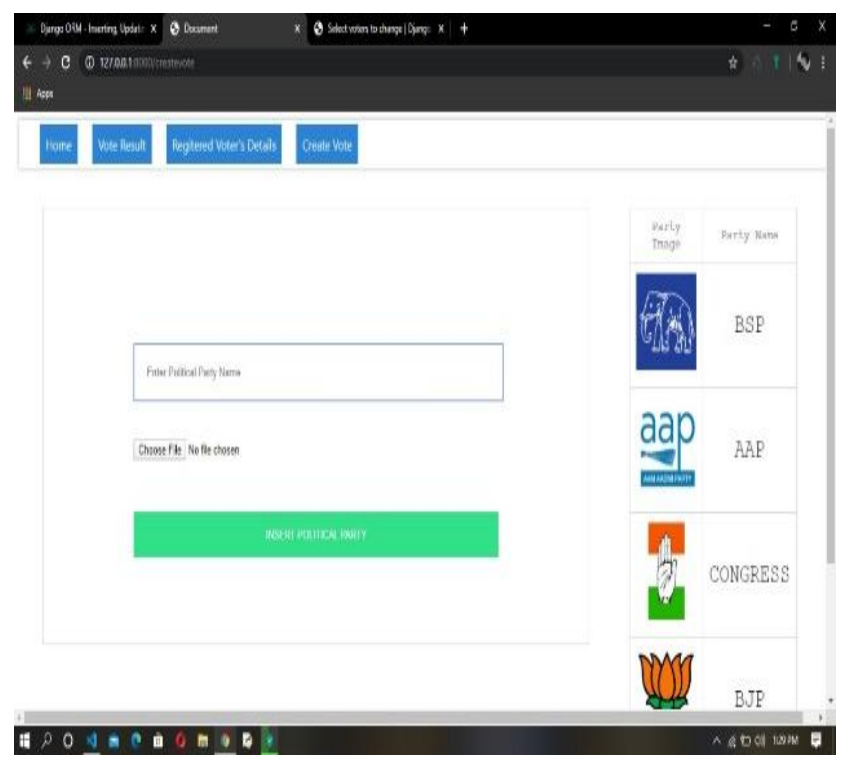

Fig 4: Political party registration page

\section{Result}

This is voting result page. Here system will automatically count the votes and create pie chart and bar chart and show the voting result.

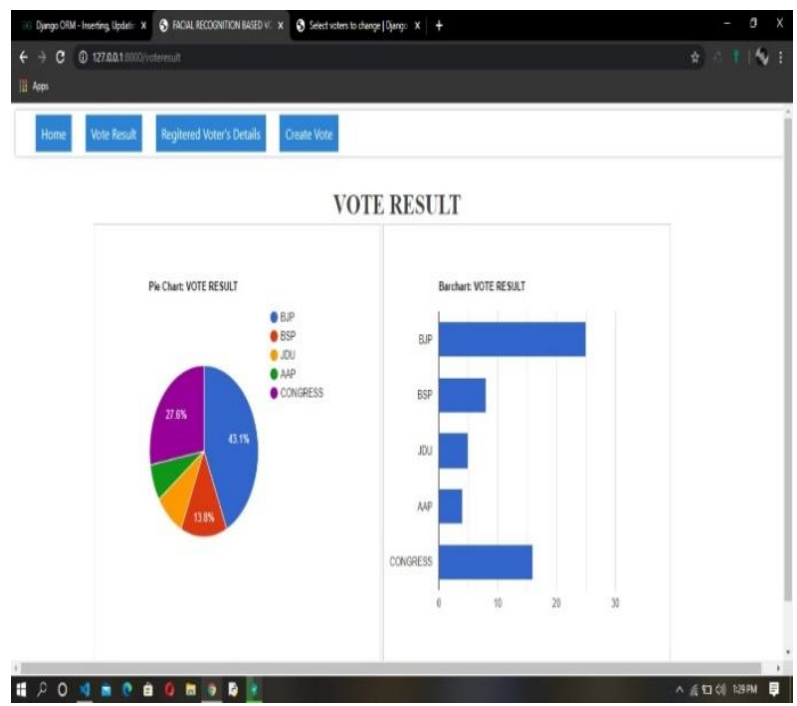

Fig 5: Result page

\section{APPLICATION}

- The main purpose of this system to make any system more safe and secure from the people who always trying to do fraud.

- We can use this system to take an attendance in colleges, corporates offices and other departments.

- We can implement this system in shopping malls or shops where we can use it to do payment.

- We can use this system to do criminal identification. This is as valuable as it is frightening, giving law authorities another method of following hoodlums the nation over.

- This can be deploy in the big server rooms where immediate action is required like airports or railway stations etc.

\section{CONCLUSION}

We have made this project to design and develop an efficient and economical system which is capable of detecting the face and test it with the database image. To make voting system more secure and providing it an online platform, this project is very beneficial. Other purpose to making this project is to save the time, money or men power and providing top class security.

\section{REFERENCE}

[1] Shrivastava, Vishesh, and Girish Tere. "An analysis of electronic voting machine for its effectiveness." International Journal of Computing Experiments (IJCE) Vol 1 (2016): 8-12.

[2] Abdulhamid, S. M., Adebayo, O. S., Ugiomoh, D. O., \& AbdulMalik, M. D. (2013). The Design and Development of Real-Time E-Voting System in Nigeria with Emphasis on Security and Result Veracity. International Journal of Computer Network and Information Security, 5(5), 9-18. https://doi.org/10.5815/ijcnis.2013.05.02 
[3] Hazzaa, F. I., Kadry, S., \& Zein, O. K. (2012). WebBased Voting System Using Fingerprint : Design and Implementation. II(Iv), 404-409.

[4] Nautiyal, J. (2013). An Automated Technique for Criminal Face Identification Using Biometric Approach. 2013(Cac2s), 608-611.

[5] Patel, C. I., \& Patel, R. (2013). Robust Face Recognition Using Distance Matrice. International Journal of Computer and Electrical Engineering, $5(4)$, 401-404. https://doi.org/10.7763/ijcee.2013.v5.740

[6]. Yamini, K., Kumar, S. M., Sonia, S., Yugandhar, P. V, \& Bharath, T. (2019). Class Attendance Using Face Detection and Recognition with OPENCV.
3822-3826.

[7]. Soomro, Z. A., \& Ali, A. (2020). FPGA based realtime face authorization system for electronic voting system.

[8]. Kavitha, S. N. (n.d.). Biometrics Secured Voting System with Finger Print, Face and Iris Verification. 743-746.

[9]. Wagner, P. (2012). Face Recognition with Python. 116.

[10]. P, J. I. P., Kishoritha, K. R., Ganesh, B., Gokulprashanth, P., \& Udhayakumar, G. (2018). Electronic Voting Machine with Facial Recognition and Fingerprint Sensors. 3, 165-170. 